\title{
José Dvoredsky: Reparando un olvido
}

\section{osé Dvoredsky: Correcting an omission}

\section{Aгq. Heгnán Munita L .}

\section{$<$ Resumen>}

El autor propone una relectura atenta de la obra de José Dvoredsky. Esta obra se enmarca en la noción de: "casas y pequeños edificios de buena calidad, dignos y sobrios, con una escala que aún hoy los hace atractivos para vivir y para recorrer, que permanecen a pesar que la idea de barrio se ha perdido y las formas de vida actual son muy diferentes de hace 50 ó 60 años". Es justamente esa postura la que nos interesa hoy en el presente número

\section{$<$ Abstract $>$}

The author of this article revisits the buildings desi gned by José Dvoredsky. These houses and small buildings are good quality si mple, worthy and dignified constructions, that in spite of the years are still attractive. No matter how much life has changed in the Iast 50 or 60 years, the idea of nei ghborhood of those years is still cherished.

<Palabras clave>

ARQUITECTURA MODERNA / ARQUITECTURA CHILENA / ARQUITECTURA RESIDENCIAL / HISTORIA DE LA ARQUITECTURA RECIENTE

$<$ Key words >

MODERNI ST ARCHI TECTURE / CHI LEAN ARCHI TECTURE I RESI DENTI AL ARCHI TECTURE / HI STORY OF CONTEMPORARY ARCHI TECTURE
Cuando recorremos la ciudad de Santiago -y también algunas importantes ciudades del paísnos Ilaman la atención algunas casas y edificios, que por lo general fueron hechas por arquitectos que dejaron su impronta y su pensamiento arquitectónico en las comunas y barrios que destacaron por su destino habitacional, como fueron en su época Ñuñoa, Providencia y más tarde La Reina y Las Condes, lo que Moreno y Eliash ${ }^{1}$ llamaron "la arquitectura doméstica, la de servicios". Estos barrios, configurados bajo el concepto de ciudad jardín, están compuestos la más de las veces por casas y pequeños edificios de buena calidad, dignos y sobrios, con una escala que aún hoy los hace atractivos para vivir y para recorrer, que permanecen a pesar que la idea de barrio se ha perdido y las formas de vida actual son muy diferentes de hace 50 ó 60 años.

Mucha de esa arquitectura fue obra de arquitectos poco conocidos ahora, a quienes hemos empezado a olvidar y que el paso del tiempo y los desarrollos inmobiliarios de los últimos años, han hecho desaparecer considerables cantidades de esas obras, que hoy recordamos con nostalgia sin habernos dado, las más de las veces, el tiempo para dejar un registro de su existencia.
José Dvoredsky fue uno de estos arquitectos, que participó de este proceso a partir de 1940. Aún hoy permanecen en buenas condiciones una parte importante de sus obras, aunque algunas han desaparecido, lentamente, transformadas en oficinas o simplemente bajo la indiferencia del negocio inmobiliario.

Desde hace casi 35 años, vive en Cincinnat este arquitecto de múltiples facetas que además de ejercer su profesión, ha hecho pintura y escultura, hizo docencia como profesor de Taller en la Escuela de Arquitectura de la Universidad de Chile, en Cerrillos, y que en su quehacer y en sus proyectos marcó un sello personal a sus obras, de tal carácter que es posible reconocer su autoría al recorrer la ciudad.

Nacido en Santiago el año en que se inicia la Primera Guerra Mundial, vive cerca del centro de Santiago y cerca del río Mapocho. Cuenta que:

“...De niño, mis padres vivían en la calle Esmeralda, entre San Antonio y Claras, frente a la Plazuela del Corregidor Zañartu y su esquina colonial que aún subsiste, frente a otra casa colonial que fue demolida para construir una residencia de estilo español, en la que, años después, el arquitecto (Eduardo) Secchi mantuvo

Arquitectura y Modernidad en Chile / 1925-1965. Una realidad múltiple. Santiago, Chile: Ediciones Universidad Católica de Chile; 15. 

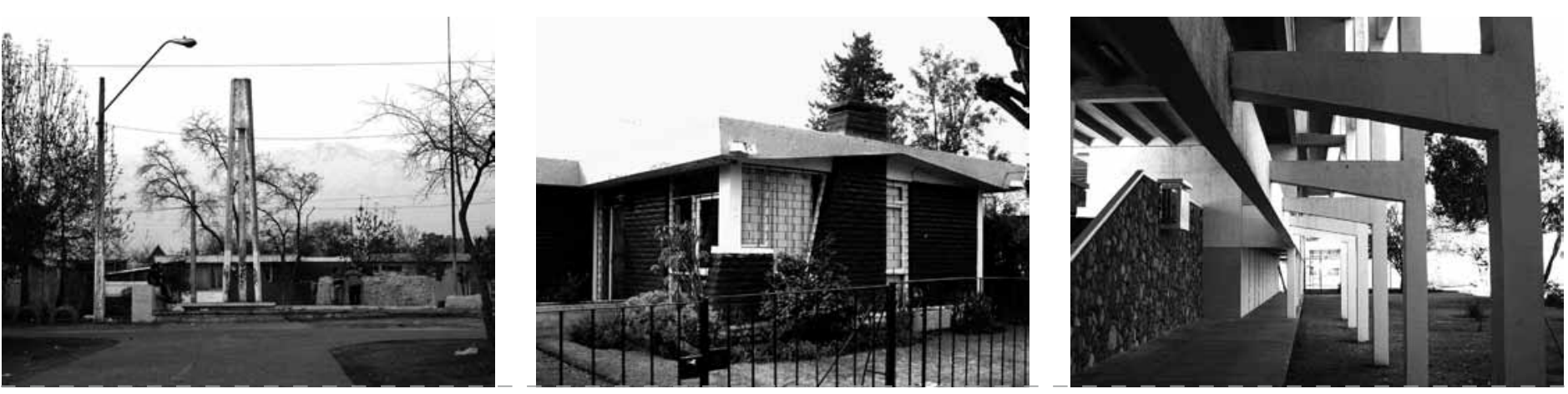

sus tertulias musicales alrededor de su magnifica discoteca".

"La calle Esmeralda de mi primera niñez, estaba empedrada con piedra de huevillo. La nueva construcción trajo el pavimento de concreto, que constituyó una bendición para nuestros patines de ruedas. [...] Patinar, subir y bajar las gradas del pórtico de la Escuela de Bellas Artes, y escalar la Fuente Alemana fueron los juegos de mi niñez".

"Mi infancia, mi adolescencia y mi juventud estuvieron ligadas al Río Mapocho, entre los puentes Recoleta y Pío Nono, con el Parque Forestal y el cerro Santa Lucia por un costado, y el barrio Recoleta y Bellavista y el cerro San Cristóbal por el otro"2.

Pertenece a una generación que le toca vivir el comienzo de la enseñanza de los postulados del Movimiento Moderno en Chile, cuando arquitectos que estudiaban o se perfeccionaban en Europa, regresan y se integran a la docencia en la Escuela de Arquitectura de la U. de Chile, ubicada en la Avenida República, en un edificio escolar de la época de Balmaceda a la que Dvoredsky ingresa en 1931. Estudia con profesores como el maestro Dávila, como conocíamos en la Escuela a don Roberto Dávila Carson, o con Rodulfo Oyarzún y Juan Martínez. Cuenta que:

"la Escuela de Arquitectura a la que ingresé, estaba imbuida del espiritu de l'Ecole de Beaux Arts de Paris. En el primer año, se dibujaban los estilos clásicos a tinta china, y después se acuarelaban. [...] En mis primeros meses de estudio de Arquitectura, aprendí a conocer los estilos clásicos, dibujándolos".

Debido a la depresión económica y a la presión de los estudiantes, se produjo la caída de Ibáñez. Toda la estructura "Beaux Arts" de la Escuela cayó también. Los alumnos de los cursos superiores pidieron tumultuosamente la Reforma Universitaria. Los profesores viejos se fueron y nuevos profesores llegaron. Ellos eran Juan Martínez, Rodulfo Oyarzún Philippi y Roberto Dávila Carson, que venían regresando de Europa, donde habían estado estudiando por su cuenta. Con ellos llegó el aire fresco de las nuevas ideas sobre Arquitectura"s.

Todo su paso por la Escuela estuvo marcado por esta impronta y la recibe y ejerce en plenitud en su vida profesional en Chile. Desarrolla desde muy temprano sus inquietudes artísticas como el dibujo y la pintura que le ayudarán a marcar un sello personal a su quehacer arquitectónico y participar de movimientos de vanguardia en Santiago, obligado a exponer en lugares no tradicionales.

"Fuimos expuestos muy tempranamente a las nuevas ideas de Europa, con anterioridad a países más poderosos. Las revistas de arquitectura europeas eran leídas ávidamente por los estudiantes y fotografías de las obras de los nuevos maestros se colgaron en los muros de la Biblioteca de la Escuela"4.

Un episodio interesante, que marca su vida universitaria y también personal, ocurre el año 1933, cuando participa en una exposición de arte para universitarios y es literalmente descubierto por Vicente Huidobro, quién lo invita junto a Waldo Parraguez a exponer en una muestra del arte de vanguardia, donde también participan sus amigas pintoras María Valencia y Gabriela Rivadeneira.

Lo que el propio Dvoredsky más destaca, es haber descubierto en Waldo Parraguez un hombre valioso y que además, sin saberlo, ambos trabajaban los mismos materiales en sus obras; Dvoredsky en la pintura y Parraguez en la escultura. Los cuadros abstractos agregaban elementos metálicos como alambres, resortes u otros objetos, también usados en las esculturas de Parraguez. Esta coincidencia los unirá el resto de los años de la Escuela, aunque Dvoredsky estaba dos cursos más abajo.
La exposición que organiza Huidobro, ocurre en el nuevo y moderno edificio Oberpaur, en forma paralela a la exposición del Salón de Arte de 1933, que se organiza en el Palacio de Bellas Artes.

Se le llamó Exposición de Diciembre y a los expositores, "decembristas", parodiando a un grupo anarquista ruso, probablemente por el marcado sello vanguardista de los expositores. Huidobro consideraba que ese era el arte que se debía hacer. Dvoredsky usa el seudónimo de Jaime Dvor, con el que aparece en todas las publicaciones de la época. La exposición causó algún revuelo y El Mercurio comenta que las obras expuestas "no corresponden a ningún género de los que el público hasta ahora conocía". Y agrega que "la exposición de los decembristas, que representa las más audaces tendencias fue indudablemente de sorpresa y desconcierto"s.

Una nueva exposición, de los mismos autores, llamada de Septiembre, la acoge la Federación de Estudiantes en su sede de Catedra 1242, con igual éxito de público, en que algunos aprueban con "entusiásticas voces de aplauso $y$ aliento, que ha merecido adversas críticas de quienes no comulgan con las tendencias allí representadas"6.

Durante la inauguración se lanza la revista de cultura Pro, a cargo de Jaime Dvor y

Eduardo Lira, quienes se autodefinen como sus "ejecutores". Sólo se publican dos números, en los que se recogen fotografías de algunos de los trabajos expuestos.

Estudió "con los nuevos profesores, sin haber tenido una experiencia previa de los estilos clásicos, con la mente limpia de reglas y convencionalismos" ${ }^{\prime \prime}$. Titulado a fines de 1939 , con un proyecto urbano de vivienda en el barrio Brasil, recibirá medalla de oro, en la exposición de proyectos de título, en el V Congreso Panamericano de Arquitectura realizado en Montevideo ${ }^{8}$ el año siguiente.

\footnotetext{
Ibid.

Ibid.

El Mercurio, 20 de diciembre de 1933; 11

El Mercurio, 14 de septiembre de 1934; 11

Ibid.

Revista Urbanismo y Arquitectura 1940; 7:24-314.
}

Carta de noviembre de 1992. Archivo arquitecto Guillermo Benavides 


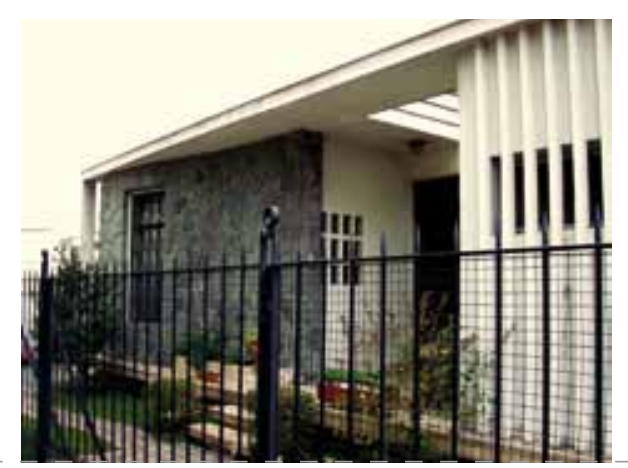

Dvoredsky será fiel en todo momento a su formación Moderna, lo que es destacado en un artículo del arquitecto Eduardo Arrau ${ }^{9}$, señalando que "el de Dvoredsky, es uno de los pocos casos en nuestro ambiente profesional en que se ha mantenido una sola línea estilística en sus realizaciones, sin hacer concesiones a la moda pasajera". Lo habitual era que quienes hacían por esos años proyectos bajo los postulados de Moderno también lo hicieran con los anteriores cánones arquitectónicos vigentes en Chile, dependiendo del cliente.

"Pertenezco a la primera generación de arquitectos con formación moderna que salió a luchar por ella y a realizarla. No fue tarea fácil. La nueva clase media alta, que prosperaba económicamente, prefería los estilos clásicos, ornamentados. El llamado estilo 'georgian' encajaba mejor con sus nuevas aspiraciones [...], Clientes más modestos, veían en esta falta de ornamentación una ventaja económica que, inconscientemente, buscaban compensar con buen diseño. Ellos fueron mis clientes preferidos. Me tocó vivir la época heroica de la Arquitectura Moderna en Chile, cuándo ésta luchaba por imponerse"10

José Dvoredsky no tuvo acceso a los grandes proyectos nacionales y sin embargo logró crear un espacio que se reconoce por sus obras, que fueron numerosas, repartidas principalmente en las comunas de Ñuñoa, Providencia, Santiago y Las Condes. Algunos de sus trabajos se encuentran también en Algarrobo, Viña de Mar, Con Con. Proyectó el Colegio Regional de La Serena para la Universidad de Chile, hoy Universidad de La Serena y el Hotel King de Arica

Sin embargo siempre fue consecuente con un pensamiento consistente, marcado por su formación en los principios del movimiento moderno, por lo que afirma que:
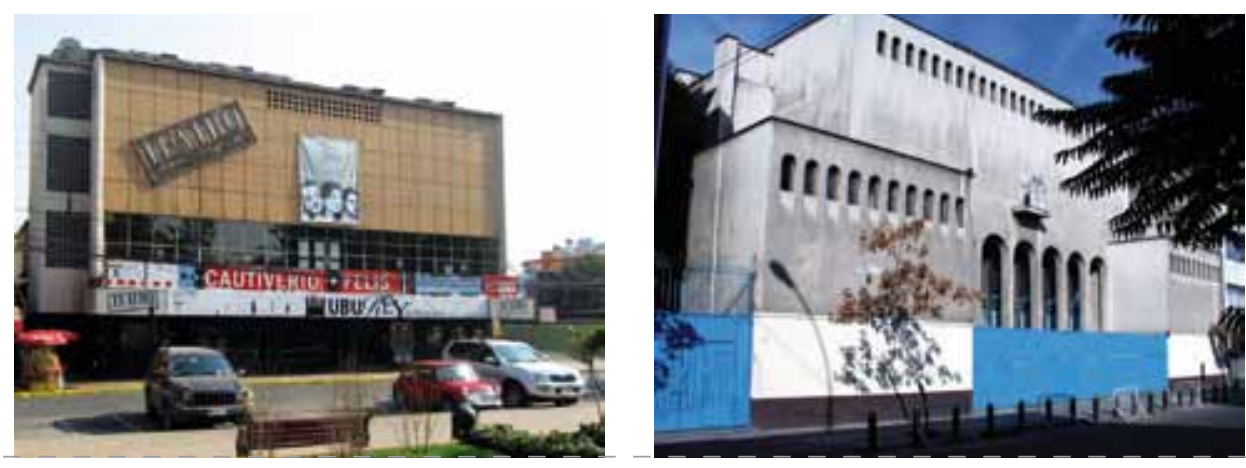

"Busqué, y busco, un equilibrio o armonía entre la racionalidad y la intuición. La expresión 'J'aime l'emotion qui corrige la règle'11 estuvo frente a mí, desde mi juventud. Fue este concepto, y la necesidad de integrar lo abstracto y lo concreto, lo que me hicieron Arquitecto, aún cuándo dibujo, pinto y hago escultura".

"La presencia de un concepto ordenador me fue siempre necesario. La Sección Áurea, como base de un sistema de proporciones, me atrajo desde mis humanidades, y la he usado siempre. Aún cuando la Sección Áurea es una sola, la forma de emplearla es personal, y agrega individualidad a los proyectos. [...] Los conceptos abstractos de dimensión, escala, proporción, orden, estuvieron siempre ligados a las muy concretas necesidades de estructura, instalaciones y edificación"12.

No estuvo ajeno a los acontecimientos de su tiempo y aunque se dedicó por entero a su trabajo, no se restó a ciertos compromisos inherentes a su postura arquitectónica. Es por eso que en 1947 suscribe, junto a una cincuentena de arquitectos nacionales un Manifiesto en que declaran estar "resueltos a desempeñar su misión profesional en concordancia con los problemas económico-sociales del país, convencidos de los ideales y principios de los congresos internacionales de arquitectura moderna

(C.I.A.M.) e inspirados en sus realizaciones..." en que reconocen el "gran contraste [...] entre los progresos alcanzados por la técnica, por la forma de la vida social, por la economía modernas y las formas de la concepción, de la realización, [...] de nuestra arquitectura y de nuestro urbanismo".

El compromiso que asumen es abocarse al "estudio del urbanismo y de la arquitectura, de acuerdo con la tónica mundial actual [...], con el fin de unificar la actividad profesional del arquitecto; de orientar las labores arquitecturales hacia las necesidades más urgentes del hombre, de incorporar estas dos disciplinas, arquitectura y urbanismo, al fenómeno del desarrollo de la vida colectiva; de enfocar los problemas de nuestra profesión a través de sus aspectos sociales y económicos; de plantear soluciones de conjunto"13.

Es por eso que declaraban:

"Que la arquitectura es el instrumento que soluciona, da forma y estructura a todos los lugares en que se desarrollan las funciones más vitales humanas".

"Que, como la economía, debe adaptarse al nombre".

"Que en ningún caso debe ser el hombre quien se adapte ni a la economía ni a la arquitectura".

"Que ni en el urbanismo ni en la arquitectura es posible abordar por separado los problemas por distintos que sean, pues todos ellos están supeditados al hombre con todo su bagaje de

"Que no es posible dar a problemas de hoy soluciones del pasado, aunque ese pasado sea ayer, porque urbanismo y arquitectura nunca pueden ser historia, sino plena actualidad"14.

Esta declaración la hacen en momentos en que el Gobierno creaba el Consejo de Economía Nacional, y que fundados en la idea de "valorizar el elemento hombre en sus aspectos espirituales y materiales, mediante la labor coordinada de los solución a la vivienda, al transporte, al trabajo a la educación, al solaz y al esparcimiento". Explican que "queremos hacer oír nuestro planteamiento, fundado -como hemos declaradoen nuestra convicción de que la economía general es la base material de nuestra sociedad, y el hombre, la medida de todas nuestras realizaciones"15. fenómenos sociales y económicos". organismos estatales ya creados con el fin de dar

Boletín del Colegio de Arquitectos 1954; 44

Carta a Paola Gianini V. y Claudia Rojas A., alumnas Universidad Mayor. Archivo arquitecto Guillermo Benavides C. Tomado de Georges Braque: "J'aime la règle qui corrige l'émotion. J'aime l'émotion qui corrige la règle" (N. del $\mathrm{A}$.). Carta citada.

Revista Arquitectura y Construcción 1947; 63.

14 ríl.

15 Ibíd. 

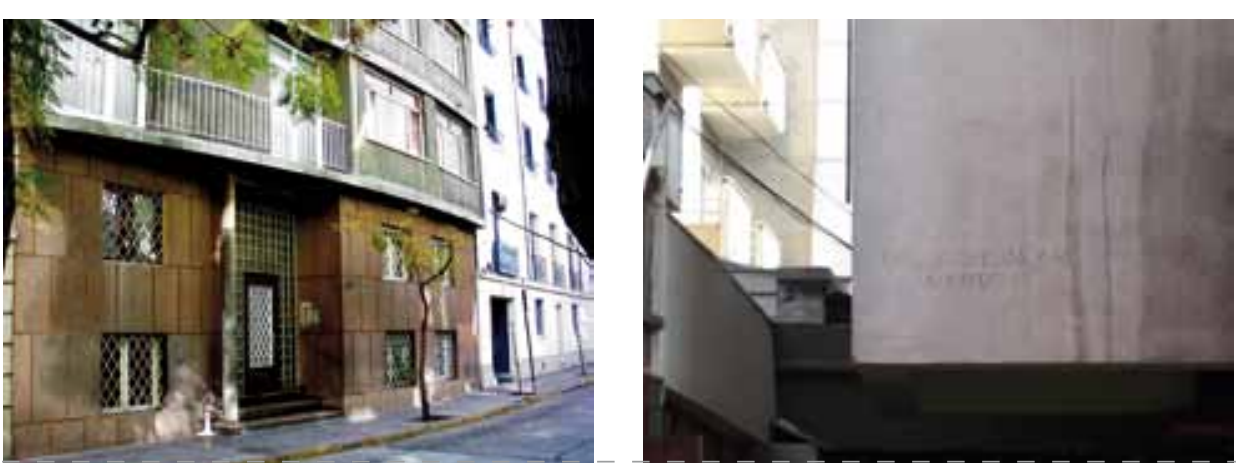

Dvoredsky manifiesta en su obra esta preocupación por el hombre, aunque no desarrolla conjuntos urbanos sino hasta unos tres lustros después. Hacia mediados de los '60, desarrolla una vivienda tipo, que denominará DVK 70, una casa para conjuntos habitacionales que construirá junto a Instituciones como Cáritas en Quilpué o con inversionistas, como en "Los Almendros de Bilbao" o en la comuna de La Reina en Echeñique 7100, en la esquina con Vicente Pérez Rosales o el "Conjunto Rubén Darío" en la calle del mismo nombre, hoy comuna de La Reina, y en La Granja, la Población Salomón Sack, que ganara por concurso, y por propia iniciativa como las casas de Lo Matta, por las que siente un especial afecto, ubicadas en terrenos que no construyó la sociedad que creó la Villa El Dorado.

En entrevista con este autor, en noviembre de 2003, en la ciudad de Cincinnati, Dvoredsky recuerda que fue uno de los pocos arquitectos que construyera casas de $70 \mathrm{~m}^{2}$, con un nivel alto de diseño y para lograr eso, en un intento que revestía un riesgo, debió asociarse con inversionistas. Detrás de estas casas reconoce que hubo un compromiso social y una búsqueda. El compromiso social estaba en darle una buena arquitectura a gente de pocos recursos. Para conseguir esto, buscó enraizarse en la tradición de la casa chilena urbana. La planta formada por dos cuadrados desfasados, "crean un vestíbulo de entrada con los servicios concentrados al frente, en una búsqueda de la economía en el diseño y la funcionalidad. Crea un patio al frente con un pequeño parrón y una puerta-ventana que daba a ese parrón o terraza con pérgola, desde un pasillo pensado como corredor o galería chilena. Los dormitorios atrás, como en aquellas casas del naranjo en el primer patio"16. Les puso chimenea que construye en parte metálica por la falta de presupuesto.

La fachada principal "es una escultura abstracta en ladrillo", con una diagonal en la chimenea, que juega dentro de dos cuadrados, en que dispone la ventana en un "muro que no es perforado sino que es una composición muro de ladrillos, lo opaco y lo transparente". La parte escultórica está en las gárgolas, con la presencia del ángulo de $45^{\circ}$, los aleros en ángulo, ambos de concreto y en algunos casos el remate de la chimenea, que también es un elemento escultórico, que juegan con las proporciones de la fachada, en que se deja entrever la razón áurea.

Buscó en una solución de pocas posibilidades, entregar todo lo que una casa de mayor superficie tiene, en que se plasma un interés arquitectónico y también social en la obra, en que "buscaba darle buena arquitectura a gente de pocos recursos". Se entiende este concepto de lo social, en que se trata de conjuntos para personas de clase media que financiaban su compra a través del desaparecido sistema de las Asociaciones de Ahorro y Préstamo, que tan útil resultó para dotar de vivienda a personas de recursos limitados pero esforzadas, que no tenían acceso a casas por intermedio de las Cajas de Previsión.

La Población Salomón Sack de La Granja, fue construida para personas de bajos recursos con el prototipo DVK 70, con un espacio público en la entrada del conjunto, en el que diseña dos agujas de hormigón que apuntan hacia la altura en homenaje a quien da el nombre al conjunto habitacional.

Una experiencia vivida cuando visitaba la obra de estas casas, lo hace recordar con orgullo y también con nostalgia aquella época. Al entrar a una de las casas, en una escalera estaba un electricista que había trabajado muchas veces con Dvoredsky y le dice desde esa posición "don José, usted es el único arquitecto con el que he trabajado que hace casas de ricos para pobres". Eso resume lo que el arquitecto buscaba con su arquitectura. abstracta en la que juegan el lleno y el vacío del

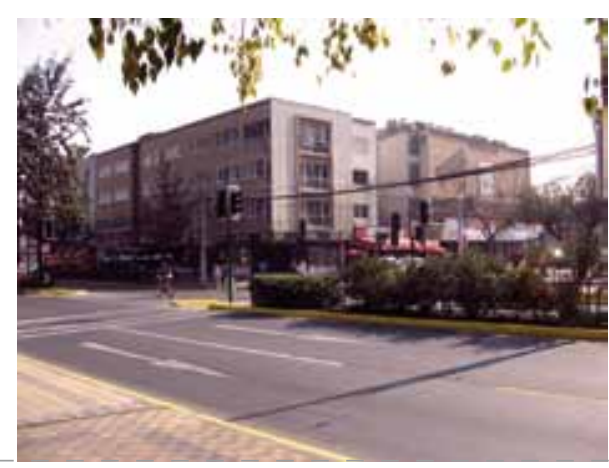

Por alrededor de una década es profesor de Taller en la Escuela de Arquitectura de la Universidad de Chile, entregando a sus alumnos su condición de hombre de valores, su bondad y atención, con una mirada sagaz, culto, en que inculca esa sensibilidad social, pidiendo que a partir de proyectos de casas sin limitaciones de superficie, se estudien nuevos proyectos, dentro del DFL 2, que contengan las mismas funciones de las anteriores, externalizando la idea social en sus alumnos.

Respecto de su paso por la Escuela como profesor, Dvoredsky dice que:

“...Durante una de las crisis de la Escuela de Arquitectura, [...] me invitaron a ser Profesor de Taller. No me imaginé, en ese momento, que estaba entrando a una de las más valiosas y hermosas etapas de mi vida. Posteriormente, en 1966, gané mi Cátedra en concurso público en examen ante la Facultad de Arquitectura y Urbanismo de la Universidad de Chile, y ante el alumnado"17.

Los que fuimos sus alumnos lo recordamos como un muy buen profesor, que nos marcó con su formación y de quien se guardan siempre buenos recuerdos. Una opción familiar lo lleva a radicarse, en 1972, en la ciudad de Cincinnati, Estado de Ohio, en los Estados Unidos, donde ejerció algunos años como arquitecto, construyendo algunas obras y dedicado a la pintura y escultura, lugar en que hoy vive retirado pero siempre interesado por la historia del Santiago fundacional, su ciudad desde la niñez.

Este recuerdo exiguo de un hombre y su obra, puede ser el inicio de otros esfuerzos para recuperar la memoria del esfuerzo y aporte hecho por tantos arquitectos de la Universidad de Chile, contemporáneos suyos, que han dejado excelentes trabajos repartidos por la ciudad y que hoy corren el riesgo de desaparecer sin dejar un adecuado registro.

\footnotetext{
16 Entrevista de noviembre de 2003.

Carta citada.
} 